\title{
Proactive Route Maintenance in Wireless Ad Hoc Networks
}

\author{
Fei Dai and Jie Wu \\ Department of Computer Science and Engineering \\ Florida Atlantic University \\ Boca Raton, FL 33431
}

\begin{abstract}
This paper presents a new routing scheme for wireless ad hoc networks that provides fresh routing information along active routes with affordable cost. The proposed routing mechanism, called proactive route maintenance (PRM), is used to replace the naive route mechanism in existing reactive (ondemand) routing protocols to improve reliability and reduce route discovery cost. The assumption behind PRM is the communication locality, i.e., most data packets are transported along a few active routes. Data packets are forwarded via multiple optimal paths to meet certain QoS requirements, such as fault tolerance and load balance. Routing information is disseminated along active routes and advertised only by active nodes that forward data packets. Alternative paths are dynamically discovered and maintained by active nodes and their 1-hop neighbors (called passive nodes). The routing overhead in passive nodes is light. PRM maintains reliable end-to-end connections in dynamic networks with low overhead, and has the desirable properties including high delivery ratio, low latency, fair load distribution, self-healing, and self-optimization. ${ }^{1}$
\end{abstract}

\section{INTRODUCTION}

Wireless network architecture can be divided into two categories [1]: the infrastructure-aided single hop model and the peer-to-peer multihop model. The former and centralized model is still dominant in wireless LANs and cellular networks. But the latter, called wireless mesh networks [2], are emerging to provide extended coverage, higher reliability, and ease of setup. An wireless ad hoc network (or simply ad hoc network) can be viewed as a special wireless mesh network, where a collection of mobile hosts forms a temporary network without the aid of any established infrastructure (i.e., base stations) or centralized administration (i.e., mobile switching centers).

Routing in ad hoc networks is challenging due to the dynamic topology and scarce resources. An ideal routing protocol should provide accurate routing information when needed, while wasting no resource in maintaining inactive routes. Most existing routing protocols are either proactive or reactive. Proactive protocols such as DSDV [3] periodically disseminate routing information over the entire network regardless of neediness and suffer from high overhead. Reactive (i.e., ondemand) protocols such as DSR [4] and AODV [5] do not update routing information unless a new path is requested (route discovery) or an old path is broken (route recovery). Route

\footnotetext{
${ }^{1}$ This work was supported in part by NSF grants CCR 0329741, ANI 0073736, and EIA 0130806. Contact address: jie@cse.fau.edu
}

discovery and recovery are usually conducted via networkwide flooding of route query (RREQ) packets, which causes high delay and control overhead. Hybrid protocols such as ZRP [6] use proactive approaches in small regions called zones and reactive approaches outside local zones. However, the zone size is either too small to provide fresh information for an active route, or too large to be cost-effective.

Multipath routing has been used in ad hoc networks to improve fault tolerance and load balance. In most existing [7], [8], [9], [10], [11], multiple paths are divided into primary (and active) paths and backup (and idle) paths. Usually, a shortest path serves as the primary path, and others become backup paths. The major drawback of these schemes is that, without active maintenance, backup paths may fail before the primary path, and switching to a failed backup path causes low delivery ratio and high end-to-end delay.

This paper proposes a hybrid routing protocol that maintains robust multipath routes with low overhead. This protocol combines reactive route discovery and proactive route maintenance (PRM) of active routes, which adapts well to highly dynamic networks and reduces the frequency of costly route recoveries. PRM provides fresh routing information at the right place with affordable cost. In PRM, multiple optimal and suboptimal paths form a mesh structure that connects source and destination. All optimal paths are active in forwarding data packets. Sub-optimal paths are backup and activated only when all optimal paths have failed. The mesh structure is selfhealing and self-optimizing. It can survive many link failures without causing route discovery or non-optimal routing. PRM is a distributed routing scheme. A freshness-based mechanism similar to those in DSDV and AODV is used to ensure loop freedom. PRM assumes communication locality: most data packets are delivered via a few active connections. Actually, communication locality is implicitly assumed by all reactive protocols. If the data traffic is distributed in many short-lived connections, the reactive protocols will be more expansive than proactive protocols.

Quality-of-Service (QoS) routing has been motivated by multimedia applications, such as voice channels, live videos, and document transfer. QoS routing selects paths based on QoS metrics to satisfy specific requirements, such as end-toend delay, delay jitter, bandwidth, and packet loss probability. In ad hoc networks, enforcing hard QoS guarantees is difficult due to the lack of a resource allocation mechanism. Most QoS 
schemes provide only soft (i.e., long-term statistical) guarantees. As PRM maintains a reliable end-to-end connection, it can be used to enhance certain soft QoS metrics such as delay, delay jitter, and delivery ratio in highly dynamic networks.

\section{RELATED WORK}

Multipath routing has been used in wired networks to achieve high throughput, load balance, and fault tolerance. Among routing protocols for ad hoc networks, TORA [12] explicitly supports multipath routing but lacks accurate distance metrics for optimal routing. MDVA [13] is designed to provide multipath routing, but its proactive manner makes it more suitable for static networks. More recently, pure reactive protocols, such as AODV [5] and DSR [4], have been extended to support multipath routing [7], [8], [9], [10], [11]. Nasipuri, Castañeda, and Das [9] suggested preserving two link-disjoint paths to the destination, at the source and each intermediate node, one as the primary path and the other as a backup. Pearlman, Haas, Sholander, and Tabrizi [10] proposed a diversity injection scheme for DSR to find node-disjoint paths. The route reply process is modified so that intermediate nodes may redirect RREPs along multiple paths back to source. Lee and Gerla [7] proposed another scheme to find maximally disjoint paths. In their split multipath routing (SMR) extension to DSR, intermediate nodes may forward, not drop, a duplicate RREQ, if this RREQ takes a route different from the previous received RREQ. Wu and Harms [11] discussed and compared both schemes. Marina and Das [8] proposed on-demand multipath distance vector routing (AOMDV), an extension to AODV. AOMDV also enable intermediate nodes to forward multiple RREPs along link-disjoint paths. An extra first hop field is added to RREQs to distinguish disjoint paths.

Multipath routing in ad hoc networks has a different set of objectives from that in wired networks. It has been shown in [10] that, due to the signal interference between multiple paths, the throughput benefit of multipath routing is trivial. Most on-demand protocol extensions [7], [8], [9] focus on fault tolerance, reducing route discovery frequency, and lower average end-to-end delay. Nasipuri et al [9] discovered that keeping a backup at each intermediate node is more fault tolerant. However, backup paths are not repaired between two route discoveries. Load balance is another concern. $\mathrm{Wu}$ and Harms [11] verified that simultaneously forwarding data packets with multiple paths improve the load balance and avoid the fast energy depletion of a few nodes.

Localized route maintenance schemes [14], [15], [16] have been designed to control the route recovery cost in reactive protocols. Castenada, Das, and Marina [16] suggested to exploit the path locality and node locality in mobile wireless networks. When recovering a broken path, the source will issue a limited flooding within a few hops around the old path. Lee and Gerla proposed AODV-BR [14], where a 1hop local repair scheme is proposed. In this scheme, nodes along the primary path overhear passing-by routing reply (RREP) packets to construct more backup paths. $\mathrm{Wu}, \mathrm{Ni}$, Tseng, and Sheu [15] proposed a similar scheme for local route recovery and optimization. All nodes along the primary path overhears both RREPs and data packets. If one node detected a better path than the current one, it will send a RREP to the upstream node, asking it to switch next hop. However, local maintenance schemes in [14], [15] use routing information collected in the last route discovery, which becomes stale quickly in a highly dynamic network. Boppana and Konduru proposed ADV [17], a DSDV-like protocol with some ondemand features. In ADV, only the routing information about active receivers (i.e., destinations of some data packets) is disseminated in the network, and the information propagation speed depends on the data traffic volume. None of these schemes uses multipath routing.

Loop-free routing is non-trivial in ad hoc networks. DSR [4] uses source routing to avoid loop, with the penalty of longer packet headers. TORA [12] uses distributed routing and is loop free, but cannot find optimal paths. In a distance vector protocol without a loop-prevention mechanism, a node with an increased distance mark may select an upstream node as its next hop. MDVA [13] use diffusion computation to avoid loop. A node with an increased distance mark cannot switch next hop until all upstream nodes have updated their distance marks. DSDV [3], AODV [5], and AOMDV [8] use destination-issued sequence number to compare the freshness of two distance marks. A node can only use a next hop with a fresher distance mark. Diffusion computation relies on the reliable hop-to-hop coordination, which is costly in ad hoc networks. The liveness of freshness-based approaches depends on the frequency that new sequence numbers are issued. DSDV uses constant frequency and has a high overhead. AODV does not issue new sequence numbers except during a route discovery. Therefore, it is hard for a node to find a new next hop. In AOMDV, a node with multiple next hops computes its distance based on the maximum distance of its next hops. It can tolerate more link failures, but causes non-optimal routing.

\section{Proactive Route Maintenance}

We assume ad hoc networks with a fixed transmitter range and bidirectional links. There is no neighbor discovery mechanism. A node is invisible to its neighbors unless it advertises its existence, but the MAC layer can detect a link failure during a unicast transmission.

\section{A. Protocol overview}

PRM can be applied to any reactive routing protocol (called the base protocol) such as DSR and AODV. Most existing protocols use a naive route maintenance mechanism. Data packets travel along a path constructed during the last route discovery. If a link failure is detected, the packet is dropped and a route error (RERR) message is sent to the source. A backup path is activated if there is one; otherwise, a route recovery is triggered. Compared with the naive route maintenance, PRM has several desirable properties:

Freshness. All nodes near an active route have the up-todate routing information. Broken paths are eliminated, new 


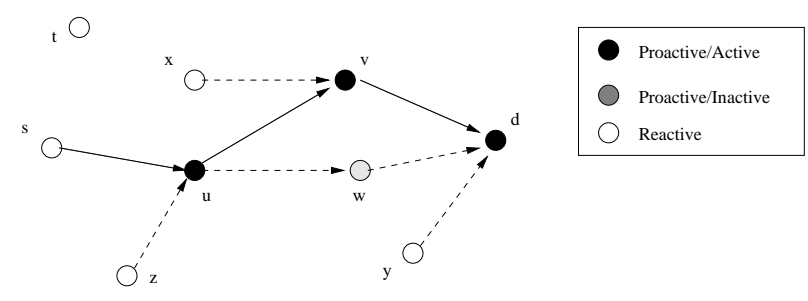

Fig. 1. An active route maintained by PRM.

paths recognized, and non-optimal paths replaced by optimal ones.

Robustness. An active node that is forwarding data packets usually maintains several fresh alternative paths. After one path fails, the data packet is usually forwarded via another path without causing packet loss or extra delay. PRM will resort to a route discovery operation only after all alternative paths have failed.

Lightweight maintenance. Unlike in existing proactive routing protocols, the route maintenance is confined to those small areas surrounding active routes, where control packets make only a small portion of data transmission. As the lifetime of a route is lengthened, the overhead of the proactive route maintenance can be compensated by the less frequent route discovery operations.

Figure 1 is a snapshot of an active route. For the sake of clarity, only one pair of source $s$ and a destination $d$ is considered in the following discussion. For each destination, a node is either in reactive or proactive mode. A reactive (white) node can forward data packets to its next hop in the base protocol, or a neighboring proactive node. Each proactive node has a watermark and forwards data packets only to other proactive nodes with lower watermarks. A proactive is active (black) if it is forwarding or receiving a certain amount of data packets per second, and inactive otherwise. An active node advertises its watermark periodically. An inactive node does not advertise its watermark unless it is within a shortest path connecting two active nodes. In Figure 1, data packets are forwarded along path $s \rightarrow u \rightarrow v \rightarrow d$. The destination $d$ and two intermediate nodes $u$ and $v$ are active nodes. The source node $s$ is a reactive node since it has not advertised its watermark yet. Node $w$ has detected an optimal path $u \rightarrow w \rightarrow d$ and advertised its watermark. It is still inactive because it has not forwarded data packets yet.

Watermarks of proactive nodes form a gradient field that attracts data packets to the destination. The destination node has the lowest watermark. The watermark of a nondestination node is computed based on neighbors' watermarks. A freshness-based mechanism similar to that of DSDV and AODV is used to ensure loop freedom in PRM. In a valid path, a previous hop always has a higher watermark than the watermark of a next hop. A node can will never raise its watermark. Therefore, a loop is impossible with monotonously decreasing watermarks.

After a route is constructed by the base routing protocol and used to forward data packets, proactive nodes emerge in the corridor area connecting the source and destination. These proactive nodes form a mesh, where each node have several alternative next hops. At each step, a random next hop is selected to forwarded a data packet. Nodes can move in and move out of the corridor without compromising the connectivity of the mesh. The overhead of advertising watermarks of proactive nodes is bounded by the size of the corridor. The corridor width depends on the traffic volume. With low traffic load, there is only a few active node at each step. The width of the corridor area, including both active nodes and inactive nodes, is small. Under heavy traffic load, previously inactive nodes will be activated to forward data packets, which in turn will solicit more inactive nodes. In both scenarios, the scalability of PRM is ensured, as the number of control packets is always proportional to the number of data packets.

\section{B. Watermark maintenance}

We use a pair (seqno, hops) as the watermark of each node, which is similar to the distance value in DSDV and AODV. Other watermark formats that support various optimizations also exist, but are omitted due to the limit of space. Here seqno is a destination-issued sequence number, and hops is its distance to the destination. Given two watermarks $w m_{i}=$ $\left(\right.$ seqno $_{i}$, hops $\left._{i}\right)$ and $w m_{j}=\left(\right.$ seqno $_{j}$, hops $\left._{j}\right), w m_{i}>w m_{j}$ if and only if seqno $_{i}<$ seqno $_{j}$, or seqno $i<$ seqno $_{j}$ and hops $_{i}>$ hops $_{j}$.

Each node $i$ maintains a list $W M$ of its neighbors' watermarks, where $w m_{j} \in W M$ represents the last announced watermark of a neighbor $j$. The watermark $w m_{i}$ of the current node $i$ is initialized to $(0, \infty)$ and evolves as follows:

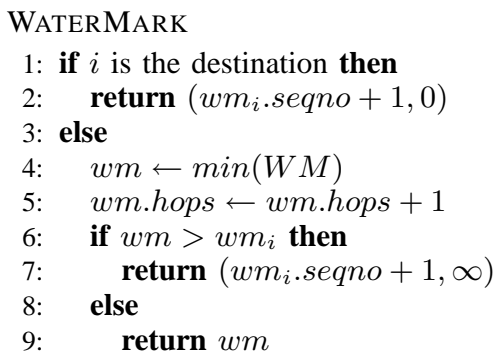

Watermark advertisement is different for active and inactive nodes. A node is active if it has sent or received at least ACTPKTNumThreSHOLD data packets during last ACTTIMEWINDOW seconds. Each active node $i$ periodically broadcast its watermark as follows:

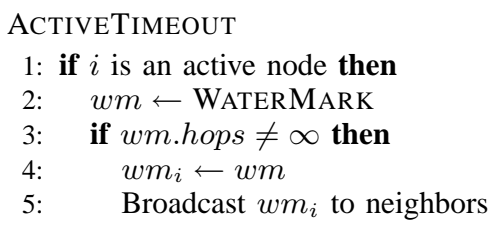

An inactive node $i$ will advertise its watermark only when it can provide an optimal path to an active neighbor $j$, as shown in the following procedure.

RECEIVENONERROR $\left(j, w m_{j}\right)$

1: Update $W M$ with $w m_{j}$ 


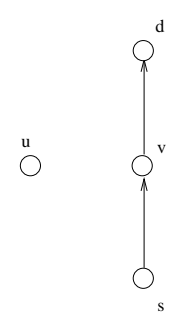

(a)

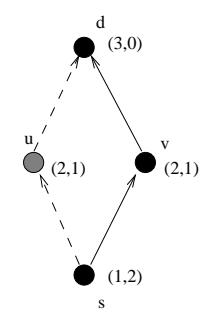

(d)

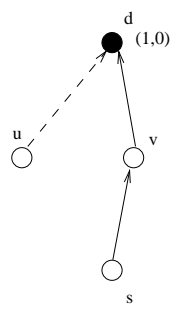

(b)

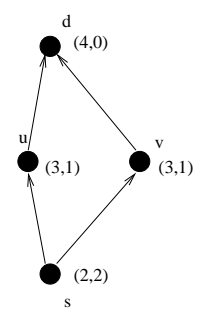

(e)

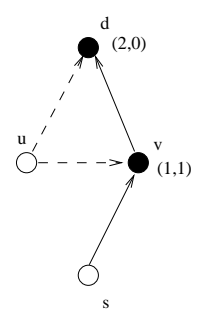

(c)

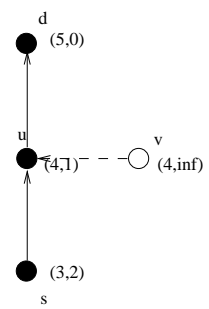

(f)
Fig. 2. A scenario study of PRM.

2: if $i$ is inactive and $j$ is active then

$w m \leftarrow$ WATERMARK

if $w m_{j} . s e q n o \leq w m . s e q n o \wedge w m_{j} . h o p s>w m . h o p s$ then $w m_{i} \leftarrow w m$

Send $w m_{i}$ to $j$

When a node $j$ receives a data packet, it will randomly select a next hop with a lower watermark to forward the packet. If no such next hop is available, it drops the packet and broadcasts an error message $\left(w m_{j}\right.$. seqno $\left.+1, \infty\right)$ to its neighbors. When a node $i$ receives this message from $j$, or a link $(i, j)$ fails, the following procedure is triggered.

\section{$\operatorname{RECEIVEERROR}(j)$}

1: Update $W M$ with $w m_{j}$

2: $w m \leftarrow$ WATERMARK

3: if wm.hops $=\infty \wedge w m_{i}$.hops $\neq \infty$ then

4: $\quad w m_{i} \leftarrow w m$

5: $\quad$ Broadcast $w m_{i}$ to neighbors

Note that when $j$ is the single next hop of $i, i$ will also broadcast an error message to its neighbors. The same procedure is repeated until an alternative path is found at an upstream node, or the source node is reached and the base protocol is invoked to discover a new route.

Figure 2 illustrates PRM in a sample network with 4 nodes. The source node $s$ and the destination node $d$ are stationary. The other nodes move from the left to the right. (a) Initially, all nodes have watermark $(0, \infty)$ and data packets are forwarded along path $s \rightarrow v \rightarrow d$, which is discovered by the base protocol. (b) After ACTTIMEWINDOW seconds, $d$ advertise its watermark $(1,0)$ to $u$ and $v$. Both $u$ and $v$ can use $d$ as the next hop. The dashed line from $u$ to $d$ indicates the potential nexthop relationship. (c) After other ACtTImeWindow seconds, $d$ issues a new sequence number. Active node $v$ computes its $w m_{v}=(1,1)$ based on last $w m_{d}=(1,0)$ and advertises $w m_{v}$ to neighbors. Both nodes $u$ and $s$ view $v$ as a next hop. (d) In the next round, active nodes $d, v$, and $s$ advertise their
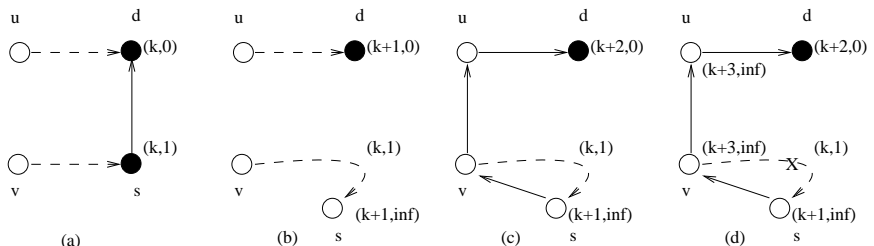

Fig. 3. Without appropriate mechanism, routing loop may emerge among reactive nodes and previously proactive nodes.

watermarks. Upon receiving watermark $w m_{s}$, inactive node $u$ determines that it can provide an optimal alternative path to $s$ and, therefore, sends $w m_{u}=(2,1)$ to $s$. Since $w m_{u} \ngtr w m_{v}$, node $u$ can no longer use node $v$ as a next hop. Meanwhile, node $s$ can use node $u$ as a next hop. (e) After receiving packets from $s, u$ becomes an active node and periodically advertise watermarks. (f) As $v$ moves rightward, $s$ detects a broken link $(s, u)$ and since then forwards data packets to node $u$ only.

\section{Loop freedom}

PRM guarantees loop freedom. Given a graph $G=(V, E)$, a directed graph $G^{\prime}=\left(V, E^{\prime}\right)$ can be induced from the next hop relationship, where a directed link $(u, v)$ exists in $E^{\prime}$ if and only if node $u$ can use node $v$ as a next hop. We say a routing protocol is loop free, if the induced graph is always a directed acyclic graph. It has been proved in [3] that a routing protocol is loop free, if it uses monotonously decreasing watermarks and each node selects only low watermark nodes as next hops. Therefore, PRM is loop free in the subnetwork consisting of proactive nodes. In the subnetwork consisting of reactive nodes, loop freedom is ensured by the base protocol. However, a loop may occur in a network with both reactive and proactive nodes.

Figure 3 shows a loop involving reactive nodes and previously proactive nodes: (a) Originally, $s$ is active, and its watermark $(k, 1)$ is overheard by a reactive node $v$. (b) After link $(s, d)$ breaks, $s$ advertises its new watermark $(k+1, \infty)$ and becomes a reactive node. However, the advertisement is lost and not heard by node $v$. (c) A new path $s \rightarrow v \rightarrow u \rightarrow d$ is discovered by the base protocol. However, node $u$ still remembers $s$ 's old watermark, and a loop is formed between nodes $s$ and $v$.

We use a simple mechanism to prevent such a loop: (1) a proactive node will not use the next hop provided by the base protocol, and (2) when a new path is discovered by the base protocol, the watermark of all involved nodes are set to $(k+1, \infty)$, where $k$ is the maximum seqno in all their previous watermarks. We define this watermark as the path low bound. As shown in Figure 3 (d), since the new watermark of node $v$ is smaller than the last heard watermark of $s$, the next hop relationship $(v, s)$ no longer exists. The following theorem guarantees loop freedom under such a mechanism.

Theorem 1: If the base routing protocol is loop free, and nodes in each path discovered by the base protocol are 
TABLE I

PRELIMINARY SimUlation RESUlT

\begin{tabular}{|c|c|c|c|c|}
\hline Protocol & $\begin{array}{c}\text { Delivery } \\
\text { Ratio }\end{array}$ & $\begin{array}{c}\text { Average } \\
\text { Delay }\end{array}$ & $\begin{array}{c}\text { Discovery } \\
\text { Overhead }\end{array}$ & $\begin{array}{c}\text { Maintenance } \\
\text { Overhead }\end{array}$ \\
\hline \hline AODV & $89.00 \%$ & $0.299 \mathrm{~s}$ & $2236 P k t$ & $0 P k t$ \\
\hline PRM & $91.29 \%$ & $0.153 \mathrm{~s}$ & $1688 P k t$ & $5033 P k t$ \\
\hline
\end{tabular}

assigned a watermark of the path low bound, then PRM guarantees loop freedom.

Proof: Suppose at one moment, a loop $v_{1} \rightarrow v_{2} \rightarrow$ $\ldots \rightarrow v_{l} \rightarrow v_{1}$ exists in the induced graph $G^{\prime}$. Because the base routing protocol is loop free, the loop contains at least one link that is directed from a high watermark node to a low watermark node. Without loss of generality, let $\left(v_{l}, v_{1}\right)$ be that link. That is, $w m_{v_{k}}>w m_{v_{1}}^{\prime}$, where $w m_{v_{1}}^{\prime}$ is the latest watermark of $v_{1}$ heard by $v_{l}$. We will show that $w m_{v_{l}} \leq$ $w m_{v_{1}} \leq w m_{v_{1}}^{\prime}$, which is a contradiction.

In the above loop, $v_{1}$ can select $v_{2}$ as its next hop, it is either because $w m_{v_{1}}>w m_{v_{2}}^{\prime}$, where $w m_{v_{2}}^{\prime}$ is the latest watermark of $v_{2}$ that is heard by $v_{1}$, or because both nodes $v_{1}$ and $v_{2}$ are reactive nodes belonging to the same path discovered by the base protocol and, hence, $w m_{v_{1}}=w m_{v_{2}}$ is the low bound of this path. In either case, we have $w m_{v_{2}} \leq w m_{v_{1}}$. Similarly, we have $w m_{v_{3}} \leq w m_{v_{2}} \leq \ldots \leq w m_{v_{k+1}} \leq w m_{v_{k}} \leq \ldots \leq$ $w m_{v_{l}} \leq w m_{v_{l-1}}$. That is, $w m_{v_{l}} \leq w m_{v_{1}}$.

\section{Simulation}

We implemented PRM on $n s 2$ [18], using AODV as a base protocol. The performance of PRM-enhanced AODV is compared with the original AODV protocol. The simulated network has 50 nodes randomly deployed in a $670 \times 670 \mathrm{~m}^{2}$ area, a uniform transmission range of $250 \mathrm{~m}$, and a bandwidth of $2 \mathrm{Mb}$. The MAC layer protocol is IEEE 802.11 . We use 10 CBR flows with 4 packet per second and 512 bytes per packet. Node movement follows the random waypoint model with a maximal speed of $10 \mathrm{~m} / \mathrm{s}$. Among the protocol parameters, ACTPKTNUmThreshold is 1 , and ACTTIMEWINDOW is $1 s$. The simulation lasts $200 s$.

Table I shows the preliminary results obtained by a single run under such configuration. Compared with the original AODV protocol, the PRM-enhanced protocol has slightly higher delivery ratio and significantly lower average end-toend delay. PRM has also lower route discovery overhead in number of control packets. However, PRM has a higher route maintenance overhead. We expect better results from the ongoing extensive simulation, where several optimization will be implemented. We also expect the performance of AODV to be worse in large and highly mobile networks.

\section{CONCLUSION}

This paper proposes a hybrid routing scheme for ad hoc networks. It uses proactive route maintenance (PRM) mechanism to replace the naive route maintenance in existing reactive routing protocols. Compared with existing local route repair and optimization schemes, PRM maintains active backup paths and adapts well to highly dynamic networks. By forwarding data on several optimal paths, PRM achieves several desirable properties, such as load balance, higher reliability, low average delay, and low delay jitter.

PRM is also scalable. Only nodes in active routes and their neighbors exchange routing information. The route advertising frequency is proportional to the traffic volume. The overhead can be further reduced by embedding route advertisement in data packets or MAC layer control packets. By exploiting communication locality and applying proactive maintenance to a confined area, we provide a new paradigm for high reliability, low overhead, multipath routing in ad hoc networks. Our future work includes a comprehensive simulation study of PRM, and variations of PRM based on more sophistical techniques.

\section{REFERENCES}

[1] Q. Xue and A. Ganz, "Qos routing in mesh-based wireless networks," International Journal of Wireless Information Networks, vol. 9, no. 3, pp. 179-190, July 2002.

[2] F. Bulk, "Understanding wireless mesh networks," Mobile Pipeline, June 2004, http://www.mobilepipeline .com/howto/21600011.

[3] C. E. Perkins and P. Bhagwat, "Highly dynamic destination-sequenced distance-vector routing (DSDV) for mobile computers," Proc. ACM SIGCOMM, pp. 234-244, 1994.

[4] D. B. Johnson and D. A. Maltz, Dynamic source routing in ad hoc wireless networks, T. Imielinski and H. Korth, editors, Mobile computing. Kluwer Academic Publishers, 1996, ch. 5, pp. 153-181.

[5] C. E. Perkins and E. M. Royer, "Ad hoc on-demand distance vector routing," Proc. IEEE WMCSA, pp. 90-100, 1999.

[6] Z. J. Haas and M. R. Perlman, "The zone routing protocol (ZRP) for ad hoc netowrks," IETF Internet Draft. http://www.ietf.org/internetdrafts/draft-ietf-manet-zone-zrp-00.txt, 1997.

[7] S. Lee and M. Gerla, "SMR: Split multipath routing with maximally disjoint paths in ad hoc networks," Proc. ICC, pp. 3201-3205, June 2001.

[8] M. K. Marina and S. R. Das, "On-demand multipath distance vector routing for ad hoc networks," Proc. ICNP, Nov. 2001.

[9] A. Nasipuri, R. Castañeda, and S. R. Das, "Performance of multipath routing for on-demand protocols in ad hoc networks," ACM/Kluwer Mobile Networks and Applications (MONET) Journal, vol. 6, no. 4, pp. 339-349, Apr. 2001.

[10] M. R. Pearlman, Z. J. Haas, P. Sholander, and S. S. Tabrizi, "On the impact of alternate path routing for load balancing in mobile ad hoc networks," Proc. ACM MobiHoc, pp. 3-10, 2000.

[11] K. Wu and J. Harms, "Multipath routing for mobile ad hoc networks," Journal of Communications and Networks, vol. 4, no. 1, pp. 48-58, Mar. 2002.

[12] V. D. Park and M. S. Corson, "A highly adaptive distributed routing algorithm for mobile wireless networks," Proc. IEEE INFOCOM, pp. 1405-1413, 1997.

[13] S. Vutukury and J. J. Garcia-Luna-Aceves, "MDVA: a distance-vector multipath routing protocol," Proc. IEEE INFOCOM, pp. 557-564, 2001.

[14] S. Lee and M. Gerla, "AODV-BR: Backup routing in ad hoc networks," ACM/Kluwer Mobile Networks and Applications (MONET) Journal, pp. 1311-1316, 2000.

[15] S.-L. Wu, S.-Y. Ni, Y.-C. Tseng, and J.-P. Sheu, "Route maintenance in a wireless mobile ad hoc network," Proc. HICSS, 2000.

[16] R. Castenada, S. R. Das, and M. K. Marina, "Query localization techniques for on-demand routing protocols for mobile ad hoc networks," ACM/Kluwer Wireless Networks (WINET) Journal, vol. 8, no. 2, pp. 137-151, Mar. 2002.

[17] R. Boppana and S. Konduru, "An adaptive distance vector routing algorithm for mobile, ad hoc networks," IEEE INFOCOM, Apr. 2001.

[18] K. Fall and K. Varadhan, The ns Manual (formerly ns Notes and Documentation), Apr. 2002, http://www.isi.edu/nsnam/ns/doc/. 